\title{
32. A Cytological Evidence for Gynogenetic Development of the Ginbuna (Carassius auratus langsdorfii)*)
}

\author{
By Yoshio OJIma and Norihide Asano \\ Department of Biology, Kwansei Gakuin University, Nishinomiya \\ (Communicated by Sajiro Makıno, M. J. A., Sept. 12, 1977)
}

The genus Carassius known by a common name of the Funa is divided into 5-subspecies in Japan: Kinbuna (Carassius auratus subsp.) ; Ginbuna (C.a. langsdorfii) ; Nigorobuna (C.a.glandcuris) ; Nagabuna (C. a. buergeri) and Gengorobuna (C. a. cuvieri).

It is remarkable that Ginbuna has a wide distribution through Japan, and that its sex ratio shows an extreme excess of females. According to Nakamura (1969), no male has so far been obtained in the Kanto district. In the Ginbuna common to the Lake Biwa, females were also extraordinarily excessive and this suggests its gynogenetic development. So far as the chromosome investigations have gone, the Ginbuna has 156 somatic chromosomes (Kobayashi, 1972). Hayashi (1976) mentioned that the somatic chromosome number of the Ginbuna was about 150 per cell was 1.5 times that of the Gengorobuna $(2 n=100)$.

The present article deals with some cytological evidence for the gynogenetic development of the Ginbuna and the behavior of the chromosomes in the polocyte formation.

Material and method. Spawned eggs of the Ginbuna from the Lake Biwa were artificially stimulated with spermatozoa of the Nigorobuna for their parthenogenetic development. Carother's fluid was used for fixation of eggs. Fixation was made at different intervals, initiating at 1 minute after insemination. The sections according to the routine paraffin method were stained with Mayer's hematoxylin-eosin. A PHA-culture method (Yamamoto et al., 1973) for cells from the renal tissue was applied for the determination of the chromosome number in the Ginbuna and Nigorobuna. For C-banding analysis, the slides were decolorized by ethyl alcohol, dipped into $0.07 \mathrm{M} \mathrm{NaOH}$ at room temperature, treated by $2 \times \mathrm{SSC}$ for $3 \mathrm{hrs}$. at $63^{\circ} \mathrm{C}$, and stained with $2 \%$ Giemsa solution at $\mathrm{pH} 7.0$, Sörensen buffer. The chromosomal movement in the course of the polocyte formation was examined in 10 reliable cells at metaphase and anaphase.

*) This work was supported by a grant-in-aid (No. 012201) for the scientific research from the Ministry of Education, Science and Culture of Japan. 
Results. 1) Non-inseminated eggs. At the time of spawning, the egg showed the metaphase spindles for the gynogenetic division (Fig. 1), since there was no evidence for the first and second meiotic divisions in them. This feature emphasizes that insemination is
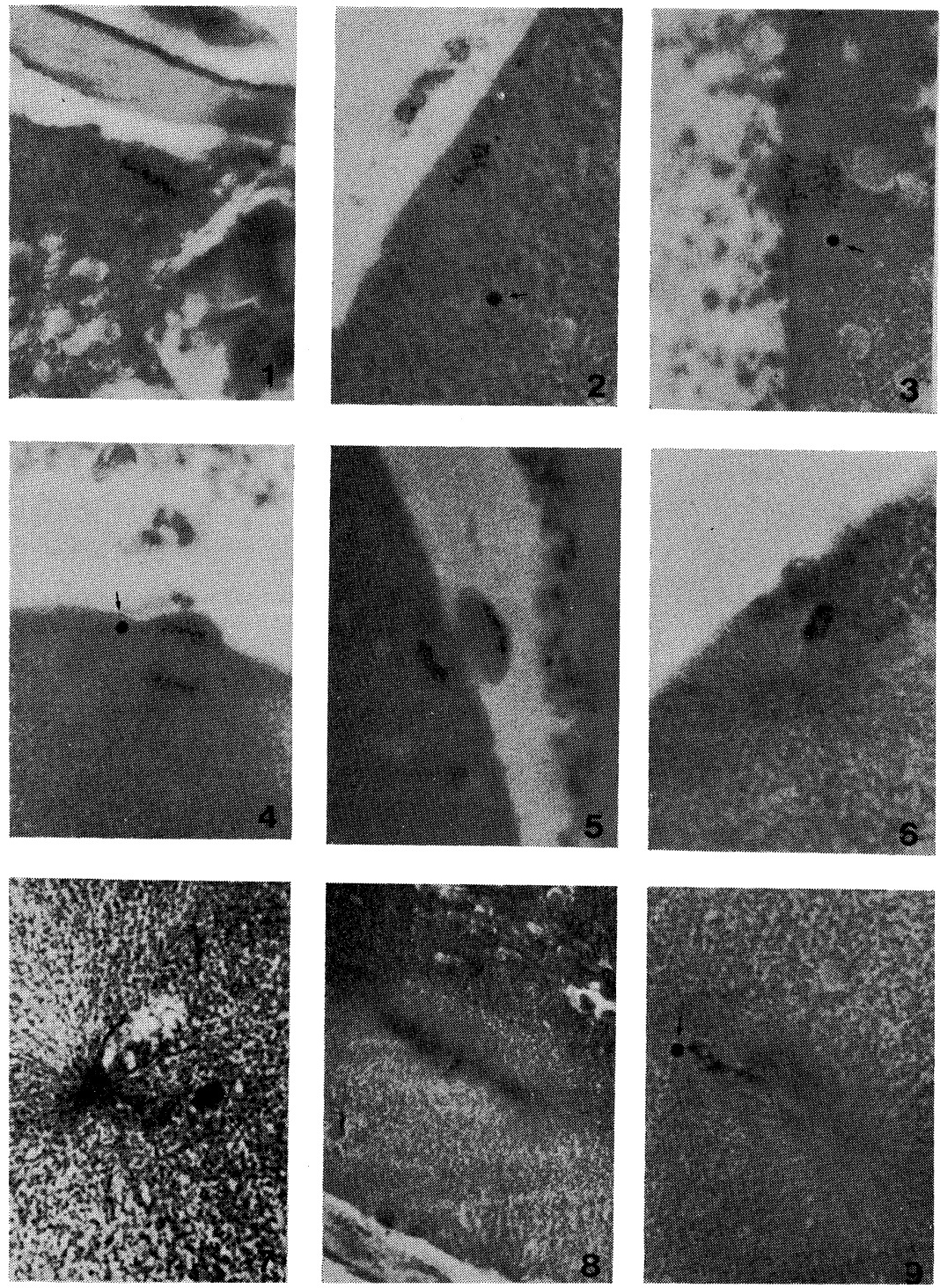

Figs. 1-9. 1: Metaphase spindle of a non-inseminated egg. 2: Metaphase spindle of an inseminated egg. Arrow shows a sperm-head. 3: Anaphase stage. Arrow shows a sperm-head. 4: Anaphase stage with developing aster. Arrow shows a sperm-head. 5: The division of the polar spindle. 6: Formation of an egg nucleus. 7: Female pronucleus. Arrow shows a sperm-head. 8: The metaphase of the first cleavage, 9: A sperm-head lying close to the egg nucleus in one blastomere. 
necessary antecedent to the gynogenetic division to advance further from metaphase to anaphase.

2) Post insemination changes in the egg. The first change observed after insemination was a structural alternation in the cortex. There was a single sperm-head in the neighborhood of the metaphase spindle, though we failed to detect the micropyle along the egg membrane (Fig. 2). By 10 to 12 minutes after insemination, the spindle of the gynogenetic development was in an anaphasic condition. A single sperm-head was found situated near the spindle with its original condensed conformation (Fig. 3). The anaphase division of chromosomes advanced successively, followed by the formation of the sperm-aster with its characteristic appearance. However, the sperm-head persisted in its unchanged original configuration for 20 minutes since its penetration (Fig. 4).

About 25 to 30 minutes after insemination, the division of the polar spindle was completed (Fig. 5). Then the daughter chromosomes clumped together to form the egg nucleus (Fig. 6). The sperm-head was found situated near the female pronucleus. About 30 to 60 minutes after insemination, the metamorphosed female nucleus appeared at the animal pole of the egg. It appeared as a spherical condensed body, a female pronucleus, lying in the naked state in the ooplasm, while the sperm-head persisted in its original form at the place of penetration. About 60 minutes after insemination, the

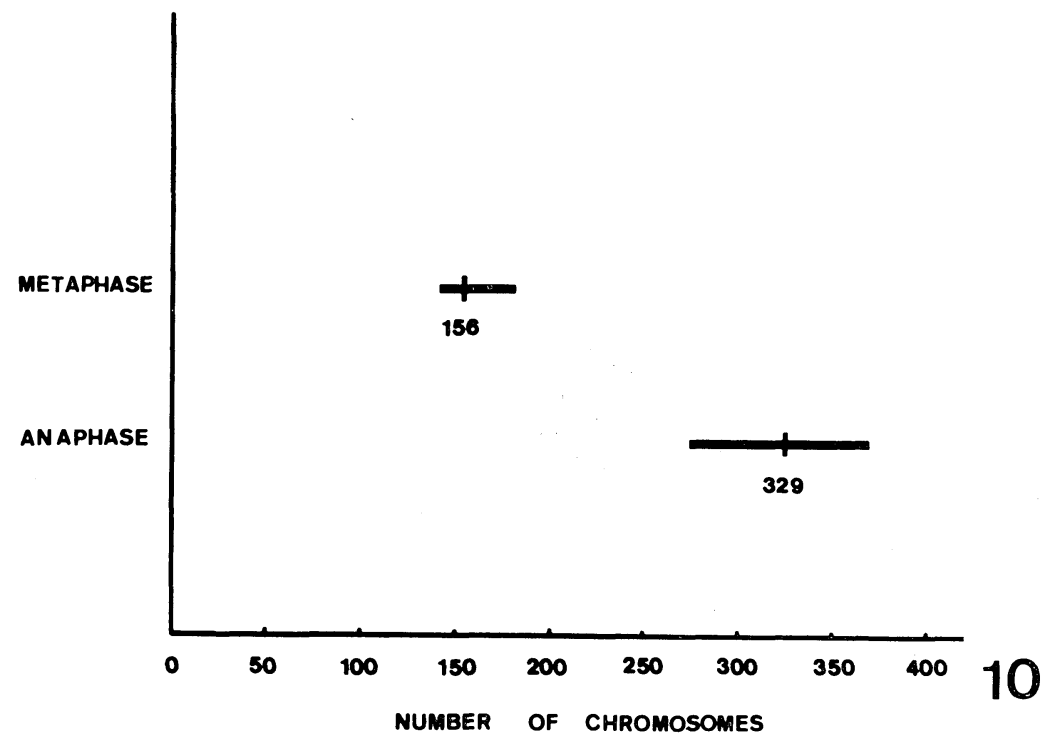

Fig. 10. Chromosomes in metaphase and anaphase spindles in the course of the polar body formation. 
female pronucleus developed spindle fibers as usual. The sperm-head remained in a still compact figure without transforming into the male pronucleus (Fig. 7). About 90 minutes after insemination, the metaphase chromosomes of the female pronucleus origin were found forming the equatorial plate of the first cleavage (Fig. 8). About 120 minutes after insemination, the metaphase plate of the second cleavage was formed. At this stage, the sperm-head was noted as a condensed body lying close to the egg nucleus in one blastomere (Fig. 9). Thus, the female pronucleus showed no slight evidence of conjugation with the regressed polar body.

3) Chromosome counts in the polar body formation. Chromosomes at metaphase and anaphase genogenetic development are shown in Fig. 10. The number of chromosomes at metaphase was approximately 150, while the anaphase ones was 300 in number.

Discussion. The Ginbuna has a wide distribution through Japan with the population consisting all of female specimens. Kobayashi (1971) reported that the Ginbuna specimens in the Kanto district were characterized by $150 \pm \sim 200 \pm$ chromosomes suggesting the gynogenetic development without regular fertilization. It is of special interest to find a classic literature at the Edo era which described a female population of the Funa from the Lake Biwa.

Our recent C-banding analysis revealed that specimens from the Lake Biwa were characterized by 153 diploid chromosomes (Hayashi unpublished).

It was shown by Ojima (1943) that the first maturation division of the carp egg was completed prior to ovulation, and that at the time of insemination the second maturation division of the egg persisted at metaphase. The second division was completed after insemination, and the formation of the second polar body followed. About 40 to 50 minutes after insemination, the metamorphosed female pronucleus appeared at the animal pole of the egg. As early as 50 to 60 minutes after sperm-entrance, the head of the spermatozoon was found completed into the spherical male pronucleus. Then, the conjugation of both pronuclei occurred at the deeper part of the animal egg pole.

As mentioned above, the sperm-head remained without transformation in the present experiment: the spermatozoon was found in situ lying close to the spindle of the nucleus. It is very probable that the sperm-head seems to be digested without offering any genetic function.

Ohno (1974) has mentioned that triploidy in connection with the parthenogenetic or the gynogenetic mode of propagation is an escape which leads to an evolutional cul-de-sac, while triploidy on 
the other occasion may serve as a stepping stone to tetraploidy, which is of evolutional significance to vertebrate evolution.

Acknowledgement. The authors are grateful to Professor Emeritus, Dr. S. Makino, M. J. A., Hokkaido University, for going over the manuscript with invaluable advice.

\section{References}

Hayashi, M., Y. Ojima, and N. Asano (1976): A cell line from teleost fishes: Establishment and cytogenetical characterization of the cells. Japan. J. Genet., $51,65-68$.

Kobayashi, H. (1971) : A cytological studies on gynogenesis of the triploid ginbuna (Carassius auratus langsdorfii). Zool. Mag., 80, 316-322.

Kobayashi, H., and H. Ochi (1972) : Chromosome study of the hybrids Ginbuna (Carassius auratus langsdorfii) $\times$ Kinbuna (C. a. subsp.) and Ginbuna $\times$ Loach (Misgurunus anguillicaudatus). Zool. Mag., 81, 67-71.

Nakamura, M. (1969) : Cyprinid Fishes of Japan. Research Institute for National Resources, Tokyo.

Ohno, S. (1974) : Animal Cytogenetics. IV. Chordata I. Gebruder Borntraeger.

Ojima, Y. (1943) : Cytological observations on fertilization in the carp, Cyprinus carpio L. Japan. J. Genet., 19, 219-228.

Yamamoto, K., and Y. Ojima (1973) : A PHA-culture method for cells from the renal tissue of teleosts. Japan. J. Genet., 48, 235-238. 